\title{
Numerical modelling of the cyclic behaviour of rock material in the context of underground pumped storage hydroelectricity
}

\author{
B. Cerfontaine, F. Collin \& R. Charlier \\ Geomechanics and Engineering Geology \\ University of Liege, Belgium
}

\begin{abstract}
The use of old mines as a reservoir in the context of pumped storage hydroelectricity rises the question of their stability especially upon cyclic loading due to filling and emptying the mines. This paper presents the formulation of a new constitutive law able to represent the inherently cyclic behaviour of rocks at low confinement. The main features of the behaviour evidenced by experiments in the literature depict a progressive degradation and deformation of the material with the number of cycles. A constitutive law based on a boundary surface model is developed. It represents the brittle failure of the material as well as its progressive degradation. It also encompasses a fatigue resistance under which no degradation of the material holds. Parameters are calibrated with respect to laboratory experiments from the literature. A parametric study is carried out to highlight the role of different parameters.
\end{abstract}

\section{INTRODUCTION}

Over the last decade, the part of renewable energy has considerably grown in the energy mix. However solar and wind energy particularly suffer from a crucial drawback. The period of maximum production does not necessarily coincide with the period of maximum consumption (Førsund 2015). The pumped-storage hydroelectricity stores energy as gravitational potential energy. Water is pumped from a lower reservoir into a higher reservoir when electric power is available at a low cost (off-peak of consumption) (Steffen 2012). During the peak of energy consumption, turbines produce energy at a higher cost, ensuring the economic viability of the system. The overall allows a storage of the energy.

The idea arose to reuse disused mines, pits and quarries as lower reservoirs for pumped-storage. Ensuring the overall stability of mine galleries and quarries is one of the main challenges for engineers. Indeed the numerous pumping and filling phases of the old galleries modify continuously the stress and pore water distributions within the surrounding rock. This results into a fatigue loading of the rock material whose resistance has been shown to be lower than the monotonic resistance (Royer-Carfagni \& Salvatore 2000, Erarslan \& Williams 2012).

This papers aims at developing a constitutive law to reproduce the fatigue behaviour of rock materials at low confinement, representative of the overall problem. The formulation proposed hereinafter is based on the bounding surface model framework already developed for sands (Taiebat \& Dafalias 2008), assuming the material is rate independent. The original model is modified to take into account the salient features of the rock behaviour upon both monotonic and cyclic loading.

Experimental results are firstly presented to introduce and detail the main features of the rock behaviour. A triaxial formulation of the constitutive law is then detailed. Finally a comparison with experimental data is provided together with a parametric study illustrating how the parameters influence the results.

\section{EXPERIMENTAL REVIEW}

\subsection{Monotonic behaviour}

Failure of brittle rock is mainly driven by the generation of micro-cracks and cracks within the rock sample. An example of such a behaviour is depicted in Figure 1 for a simple compression experiment. Following the pioneering work of (Bieniawski 1967a, Bieniawski 1967b) the brittle behaviour of rocks is classically delineated into five phases for simple compression tests.

(I) The first phase corresponds to the closure of the microcracks initially existing within the rock sample. Acoustic emissions (AE) recorded in (Cai, Kaiser, Tasaka, Maejima, Morioka, \& Minami 2004) correspond to sound waves emit- 
ted when microcracks or fractures are created or propagated. During this first phase, the number of $\mathrm{AE}$ is almost equal to zero. It lasts up to a vertical stress equal necessary to ensure the crack closure $\sigma_{c c}$.

(II) The second phase is purely elastic. There is no initiation of cracks nor propagation. There are still no $\mathrm{AE}$ and the volumetric deformation is equal to the elastic one.

(III) From a vertical stress equal to $\sigma_{i}$, microcracks initiate or propagate and are mainly parallel to the applied stress. They induce a volumetric dilation. The number of acoustic emissions rises up but remains limited. This phase is termed "stable crack growth".

(IV) During the last phase, the coalescence of all microcracks takes place at $\sigma_{d}$ around 0.7 to 0.8 times the peak strength (Bieniawski 1967a, Martin 1997, Cai, Kaiser, Tasaka, Maejima, Morioka, \& Minami 2004). The dilation strongly increases as well as the number of AE.

(V) Finally failure is reached at $\sigma_{f}$. The rock sample may exhibit shear bands or macrocracks.
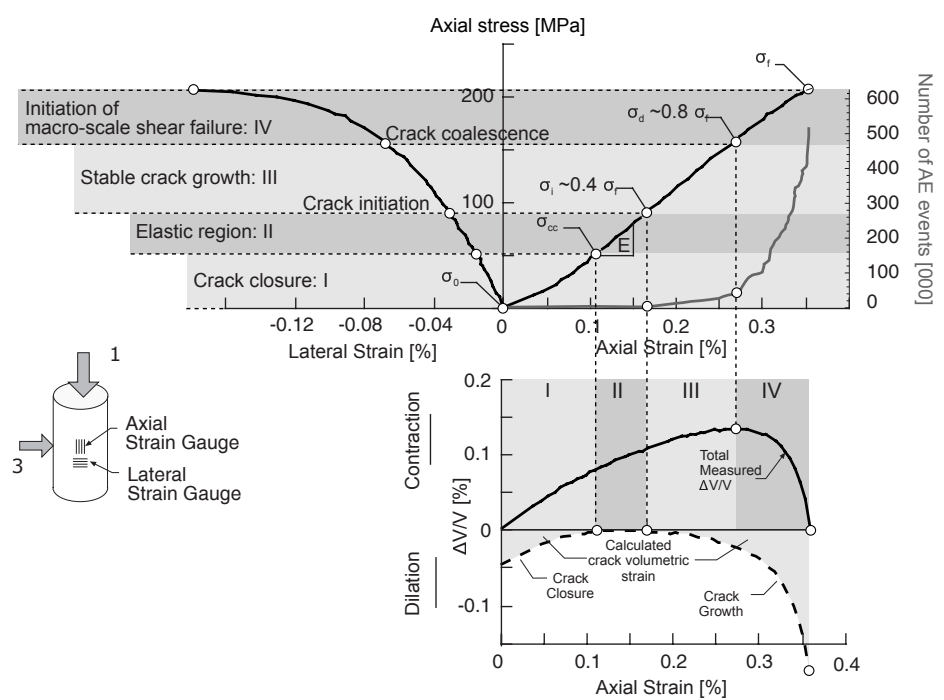

Figure 1: Stress-strain diagram exhibiting the different stages of the monotonic behaviour of brittle rocks, (Martin 1997, Cai, Kaiser, Tasaka, Maejima, Morioka, \& Minami 2004)

The brittle behaviour of rock is not limited to the simple compression experiments. It is observed also when the sample is subjected to a confinement as shown in Figure 2. It is known that the ductility increases with confinement (Liu \& He 2012). The failure may become totally ductile at very high confinement. However this paper will focus on low confinement behaviours. Many other features are not illustrated here but are relevant for the formulation of a constitutive law such as the material (Attewell \& Farmer 1973) or load-induced anisotropy (Benz \& Schwab 2008).

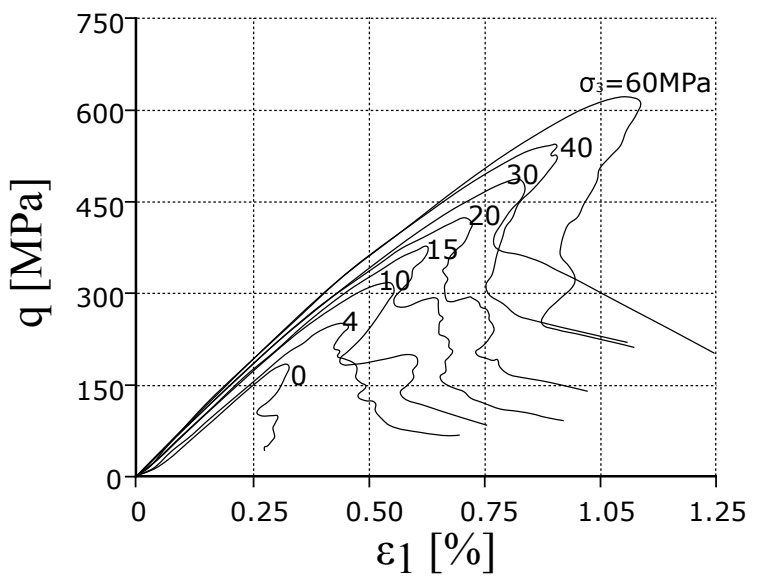

Figure 2: Brittle failure in Lac du Bonnet granite, (Martin 1997)

\subsection{Cyclic behaviour}

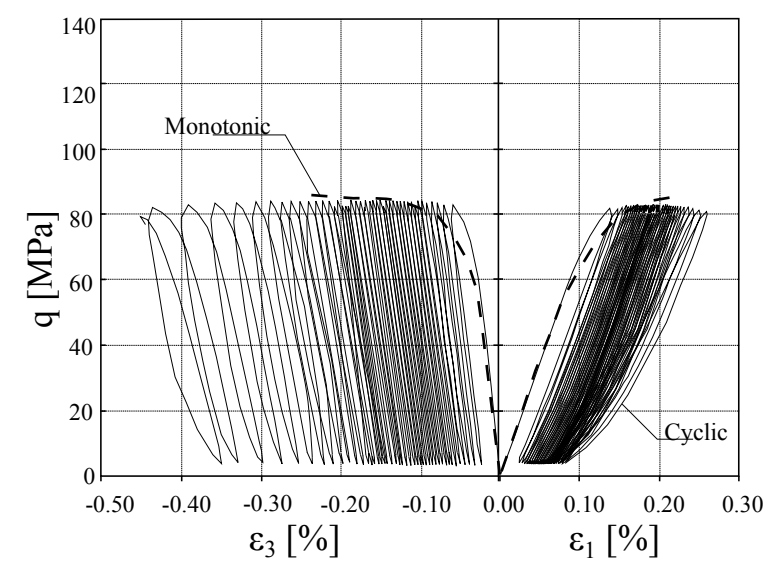

Figure 3: Monotonic and cyclic simple compression (constant amplitude) tests on Lorano marble, data traced from (Royer-Carfagni \& Salvatore 2000)

The typical behaviour of a sample of Lorano marble is illustrated in Figure 3 for both monotonic and constant cyclic amplitude experiments. The monotonic failure is brittle and presents more plastic deformation laterally than vertically. The cyclic experimental results highlight an accumulation of plastic deformation cycle after cycle. The increment of deformation increases progressively and cycles are more open at the end.

A cyclic indirect tensile test on Brisbane tuff is provided in Figure 4. This test consists in a ramp signal, i.e. the load oscillates between an increasing average load. It is observed in (Erarslan \& Williams 2012) that the cyclic resistance is systematically lower than the monotonic one. Moreover the modes of failure are different. The monotonic samples present a straight crack while cyclically loaded samples are surrounded by debris and crushed rock material. This suggests that much more microcracks are generated.

It is finally observed that upon constant amplitude cycles, a threshold exists below which there is no failure of the material even upon a large number of cycles (Erarslan \& Williams 2012). Figure 5 presents the number of cycles as a function of the amplitude applied. When the amplitude is below $70 \%$ of the 


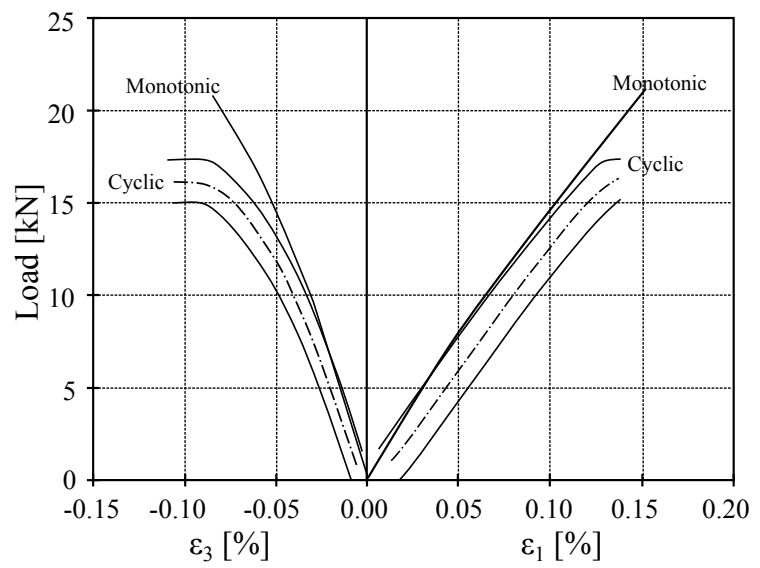

Figure 4: Comparison of monotonic and cyclic indirect tensile strength results of Brisbane tuff, (Erarslan \& Williams 2012)

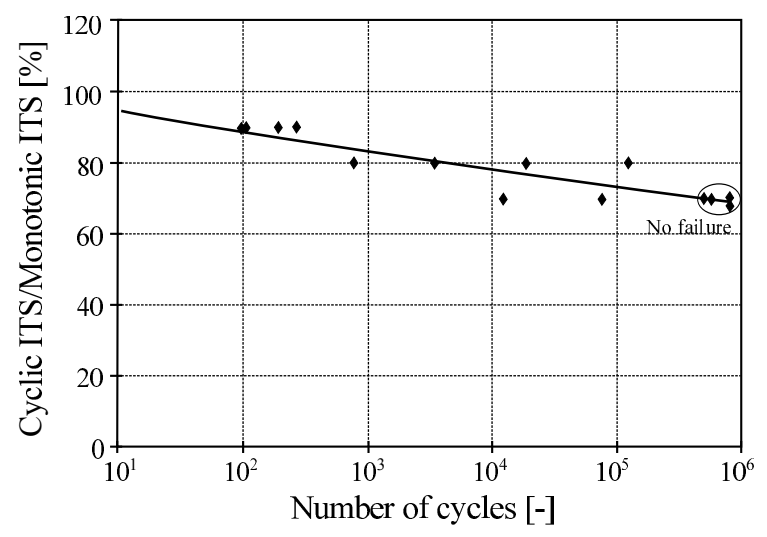

Figure 5: S-N curve for indirect tensile strength of Brisbane tuff, (Erarslan \& Williams 2012)

monotonic resistance, no failure was observed even after more than 500000 cycles. This threshold may be defined as the fatigue resistance of the material.

\section{TRIAXIAL FORMULATION OF A CONSTITUTIVE LAW}

\subsection{Triaxial notations}

In triaxial conditions, only the diagonal components of the deformation $\epsilon$ and stress $\sigma$ tensors are different from zero. Two stress invariants describes the stress state of the material

$$
\begin{aligned}
& \mathrm{p}=\left(\sigma_{1}+2 \sigma_{3}\right) / 3, \\
& \mathrm{q}=\sigma_{1}-\sigma_{3},
\end{aligned}
$$

where $\sigma_{1}$ and $\sigma_{3}$ are respectively the vertical and lateral effective stresses applied to the sample. The stresses are gathered into the vector $\mathbf{s}^{T}=\left[\begin{array}{ll}\mathbf{q}, & \mathrm{p}\end{array}\right]$ where ${ }^{T}$ is the transpose operator. A reduced deviatoric stress $\xi$ is defined according to

$$
\xi=\frac{\mathrm{q}}{\mathrm{p}+\mathrm{p}_{c}}
$$

where $\mathrm{p}_{c}$ will be further defined. Similarly strain invariants are defined according to

$\epsilon_{v}=\epsilon_{1}+2 \epsilon_{3}$,

$\epsilon_{q}=\epsilon_{1}-\epsilon_{3}$,

where $\epsilon_{1}$ and $\epsilon_{3}$ are the vertical and lateral deformations. They are assembled into the vector $\mathbf{e}^{T}=$ $\left[\epsilon_{q}, \epsilon_{v}\right]$.

\subsection{Yield, hardening and other surfaces}

The model is defined by different surfaces in the $p-q$ plane. Only the surfaces on the compression side are presented in Figure 6 for clarity. Their common apex is located on the p-axis at coordinate $-\mathrm{p}_{c}$ which is a function of the cohesion $c$ and the friction angle $\phi$

$\mathrm{p}_{c}=\frac{c}{\tan \phi}$

where $\phi$ is the friction angle.

The yield surface has an opening of $2 \mathrm{M}^{y}$. Its mathematical formulation reads

$f^{y} \equiv\left(q-\left(\mathrm{p}+\mathrm{p}_{c}\right) \alpha\right)^{2}-\left(\mathrm{p}+\mathrm{p}_{c}\right)^{2}\left(\mathbf{M}^{y}\right)^{2}$

where $\alpha$ is termed the back-stress tensor of the surface. It describes the variation of its position and is limited by

$\alpha+\mathrm{M}^{y} \leq \mathrm{M}^{b} \quad$ in compression,

$\alpha-\mathbf{M}^{y} \geq \mathbf{M}^{b} / \delta^{b} \quad$ in extension,

where $\mathbf{M}^{b}$ is the slope of the bounding surface and $\delta^{b}$ is the ratio between the compression and extension openings of the bounding surface. The slope $\mathbf{M}^{b}$ is related to the friction angle through

$\mathbf{M}^{b}=\frac{6 \sin \phi}{3-\sin \phi}$.

The yield surface is then a function of two internal variables: $\alpha$ and $\mathrm{p}_{c}$. The bounding surface is described by

$f^{b} \equiv \mathrm{q}^{2}-\left(\mathrm{p}+\mathrm{p}_{c}\right)^{2}\left(\mathrm{M}^{b}\right)^{2}$

and depends only on the internal variable $\mathrm{p}_{c}$.

The last two surfaces are termed hardening (opening $\mathrm{M}^{h}$ ) and decohesion of (opening $\mathbf{M}^{p c}$ ) surfaces. They are homothetic to Equation (11).

The purpose of the hardening surface is to allow a brittle failure of the material. Indeed in the bounding surface models (Taiebat \& Dafalias 2008), the plastic modulus is a function of the distance between the yield and the bounding surface $\alpha+\mathbf{M}^{y}-\mathbf{M}^{b}$. When those surface are very close, the plastic modulus tends 


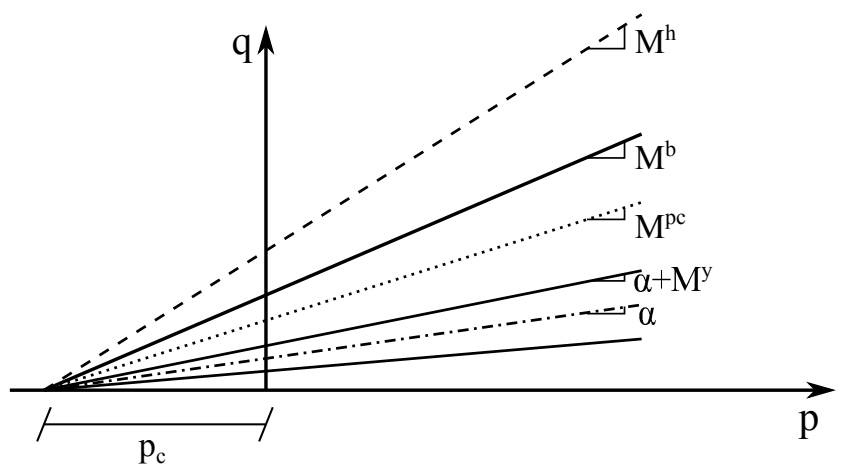

Figure 6: Description of the different surfaces of the model (compression side): $\alpha$ back-stress tensor, $\mathbf{M}^{y}$ yield surface, $\mathbf{M}^{b}$ bounding surface, $\mathbf{M}^{h}$ hardening surface, $\mathbf{M}^{p c}$ decohesion surface

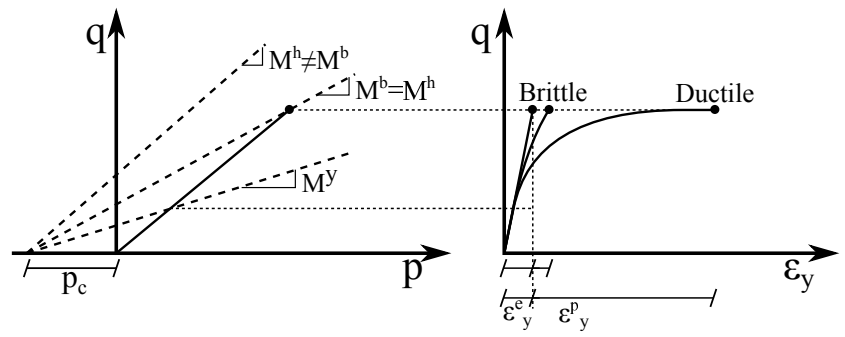

Figure 7: Difference between ductile and fragile failure

to zero and the failure is very ductile as shown in Figure 7. If the plastic modulus is computed with respect to the hardening surface $\mathbf{M}^{h} \geq \mathbf{M}^{b}$, the bounding surface is reached before the plastic modulus is set to zero and the failure is brittle.

The decohesion surface of slope $\mathbf{M}^{p c}$ defines the fatigue resistance of the rock as a function of its confinement. If the stress path lies within the decohesion surface, there is no decrease of the cohesion variable $\mathrm{p}_{c}$.

\subsection{Yield surface}

The yield surface is activated in both monotonic and cyclic loading, before failure. It is subjected to kinematic (on $\alpha$ ) and isotropic (on $\mathrm{p}_{c}$ ) hardening.

\subsubsection{Flow rule}

The flow rule is associated in the deviatoric direction and non-associated in the volumetric direction, which is classical in many cyclic constitutive laws. The variations of the plastic components of deformation is defined such that

$\dot{\mathbf{e}}^{p}=\left[\begin{array}{c}\dot{\epsilon}_{q}^{p} \\ \dot{\epsilon}_{v}^{p}\end{array}\right]=\dot{\lambda}\left[\begin{array}{c}\xi \\ A_{d} \xi\end{array}\right]=\dot{\lambda} \mathbf{G}$

where $\dot{\lambda}$ is the variation of the plastic multiplier and $A_{d}$ is a parameter of dilatancy.

\subsubsection{Hardening of internal variables}

The kinematic hardening law of the yield surface reads

$\dot{\alpha}=\dot{\lambda} h\left(\alpha^{h}-\alpha\right)\left|\alpha^{h}-\alpha\right|^{N-1}=\dot{\lambda} h_{1}$,

where $N$ is a parameter that rules the stiffness and brittleness of the material, and $\mathrm{h}$ is defined as (Taiebat \& Dafalias 2008)

$$
h=\frac{b_{0}}{\left(\mathbf{M}^{h}\left(1+1 / \Delta^{b}\right)-2 \mathbf{M}^{y}-\left(\alpha^{h}-\alpha\right)\right)^{2}},
$$

where $b_{0}$ is a material parameter. The definition of the $\alpha^{h}$ variable depends on the loading or unloading

$$
\begin{array}{ll}
\alpha^{h}=\mathrm{M}^{h}-\mathrm{M}^{y} & \mathrm{q}-\left(\mathrm{p}+\mathrm{p}_{c}\right) \alpha>0, \\
\alpha^{h}=-\mathbf{M}^{h} / \delta^{b}+\mathbf{M}^{y} & \mathrm{q}-\left(\mathrm{p}+\mathrm{p}_{c}\right) \alpha<0 .
\end{array}
$$

Such a definition allows different stiffness in loading and unloading.

The isotropic hardening rule simply reads

$$
\dot{\mathrm{p}}_{c}=\dot{\lambda} A_{c}\left(\mathrm{p}_{r e s}-\mathrm{p}_{c}\right)\left\langle\left(\alpha-\alpha^{p c}\right) \frac{\mathrm{q}}{|\mathrm{q}|}\right\rangle=\dot{\lambda} h_{2},
$$

where $A_{c}$ is a parameter ruling the rate of decohesion, the operator $\langle X\rangle=0.5(|X|+X)$ is the Mc Cauley brackets and

$$
\begin{array}{ll}
\alpha^{p c}=\mathbf{M}^{p c}-\mathbf{M}^{y} & \mathbf{q}>0, \\
\alpha^{p c}=-\mathbf{M}^{p c} / \delta^{b}+\mathbf{M}^{y} & \mathbf{q}<0 .
\end{array}
$$

The hardening laws are written in compact form such that

$\dot{\mathbf{v}}=\left[\begin{array}{c}\dot{\alpha} \\ \dot{\mathrm{p}}_{c}\end{array}\right]=\dot{\lambda}\left[\begin{array}{l}h_{1} \\ h_{2}\end{array}\right]=\dot{\lambda} \mathbf{H}$.

\subsubsection{Plastic multiplier}

Assuming the classical additive decomposition of deformation between plastic and elastic components (Simo \& Hughes 1998), the triaxial stress-strain relation is written

$\dot{\mathbf{s}}=\mathbf{E} \cdot\left(\dot{\mathbf{e}}-\dot{\mathbf{e}}^{p}\right)$

where

$\mathbf{E}=\left[\begin{array}{cc}2 \mathrm{G} & 0 \\ 0 & \mathrm{~K}\end{array}\right]$

where $\mathrm{G}$ is the shear modulus and $\mathrm{K}$ the bulk modulus. The consistency condition is then written

$\dot{f}^{y} \equiv\left[\frac{\partial f^{y}}{\partial \mathbf{s}}\right]^{T} \cdot \dot{\mathbf{s}}+\left[\frac{\partial f^{y}}{\partial \mathbf{v}}\right]^{T} \cdot \dot{\mathbf{v}}=0$ 
Inserting Equations (12), (20) and (21) into Equation (23) leads to the following expression of the plastic multiplier

$\dot{\lambda}=\frac{\left[\frac{\partial f}{\partial \mathbf{s}}\right]^{T} \cdot \mathbf{E} \cdot \dot{\mathbf{e}}}{\left[\frac{\partial f}{\partial \mathbf{s}}\right]^{T} \cdot \mathbf{E} \cdot \mathbf{G}-\left[\frac{\partial f}{\partial \mathbf{v}}\right]^{T} \cdot \mathbf{H}}$

where

$\left[\frac{\partial f}{\partial \mathbf{s}}\right]^{T}=\left[\begin{array}{ll}\frac{\partial f^{y}}{\partial q} & \frac{\partial f^{y}}{\partial p}\end{array}\right]$

and

$\left[\frac{\partial f}{\partial \mathbf{v}}\right]^{T}=\left[\begin{array}{ll}\frac{\partial f^{y}}{\partial \alpha} & \frac{\partial f^{y}}{\partial \mathrm{p}_{c}}\end{array}\right]$

\subsection{Bounding surface}

When the yield surface reaches the bounding one, the second mechanism is activated. The kinematic hardening of the yield surface is stopped since it has reached its limit, i.e. $\dot{\alpha}=0$. However the cohesion continues to degrade but at a higher rate. The cohesion degradation is similar to Equation (17) but the parameter $A_{c}$ is replaced by $A_{c 2}$ such that

$\dot{\mathrm{p}}_{c}=\dot{\lambda} A_{c 2}\left(\mathrm{p}_{r e s}-\mathrm{p}_{c}\right)\left\langle\left(\alpha-\alpha^{p c}\right) \frac{\mathrm{q}}{|\mathrm{q}|}\right\rangle$.

Moreover the derivatives of $f^{y}$ in the plastic multiplier definition, Equation(24), are replaced by derivatives with respect to $f^{b}$.

\section{EXAMPLES OF SIMULATIONS}

\begin{tabular}{cccc}
\hline \multirow{2}{*}{ Elasticity } & $\mathrm{M}^{y}[-]$ & $\mathrm{E}[\mathrm{GPa}]$ & $\nu[-]$ \\
& 0.1 & 70 & 0.16 \\
\cline { 2 - 4 } Bounding & $\mathrm{M}^{b}[-]$ & $\mathrm{p}_{\text {res }}[\mathrm{MPa}]$ & $\delta_{b}[-]$ \\
& 1.4 & 5 & 1 \\
\cline { 2 - 4 } Decohesion & $\mathrm{M}^{p c}[-]$ & $A_{c}[-]$ & $A_{c 2}[-]$ \\
& 0.5 & 20 & 500 \\
\cline { 2 - 4 } Hardening & $\mathrm{M}^{h}[-]$ & $\mathrm{Nexp}[-]$ & $\mathrm{b}_{0}[-]$ \\
& 1.5 & 2 & $3 \cdot 10^{4}$ \\
\cline { 2 - 4 } Volumetric & $A_{d 0}[-]$ & & \\
& -1.8 & & \\
\hline
\end{tabular}

Table 1: Parameters of the model

Comparison with experimental results are based on results published in the literature for the Lorano marble material. The simple compression monotonic and cyclic stress paths are presented in (RoyerCarfagni \& Salvatore 2000). Elastic and failure parameters are extrapolated from (Cattaneo \& Labuz 2001, Stavropoulou, Liolios, \& Exadaktylos 2004,

\begin{tabular}{cc}
\hline$\alpha_{0}[-]$ & $\mathrm{p}_{c 0}[\mathrm{MPa}]$ \\
0 & 34 \\
\hline
\end{tabular}

Table 2: Initial internal variables

Ferrero, Migliazza, \& Spagnoli 2009).

Parameters and initial internal variables resulting from the calibration process are provided in Tables 1 and 2. The following sections compares experimental results and numerical simulations. A parametric study is also carried out to highlight the main role of some parameters.

\subsection{Comparison with experimental data}

Results of monotonic simulations are compared with experimental data in Figures 8 and 9. Elastic parameters as well as friction angle and cohesion were estimated from the literature. Indeed triaxial tests at different confinement pressures would have been necessary.

The brittle failure is well captured in terms of both peak resistance $(\sim 85 \mathrm{MPa})$ and maximum deformation $(\sim 0.22 \%)$. The behaviour of the material was set identical in compression and extension since no data are available in extension.

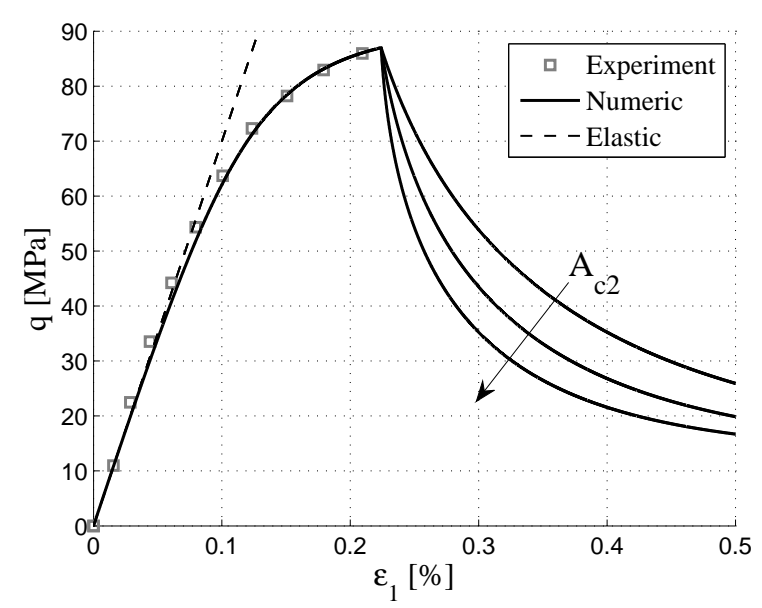

Figure 8: Comparison of monotonic simple compression simulation and experimental results, stress deviator vs. axial deformation

The post-peak behaviour of the samples was not investigated in (Royer-Carfagni \& Salvatore 2000). However according to other studies the unconfined failure is probably brittle (Cattaneo \& Labuz 2001). A steep post-peak behaviour was assumed with a slow but non zero cohesion residual parameter. It is shown in Figure 8 for different values of parameter $A_{c 2}$.

The dilatancy parameter is calibrated with respect to the volumetric deformation results presented in Figure 9. Only the pre-peak part of the results is exhibited to prove the good agreement of simulation results with experiments.

The evolution of the $\mathrm{p}_{c}$ internal variable is provided in Figure 10. The pre-peak variation of the cohesion $\mathrm{p}_{c}$ is very limited. It steeply decreases afterwards due 


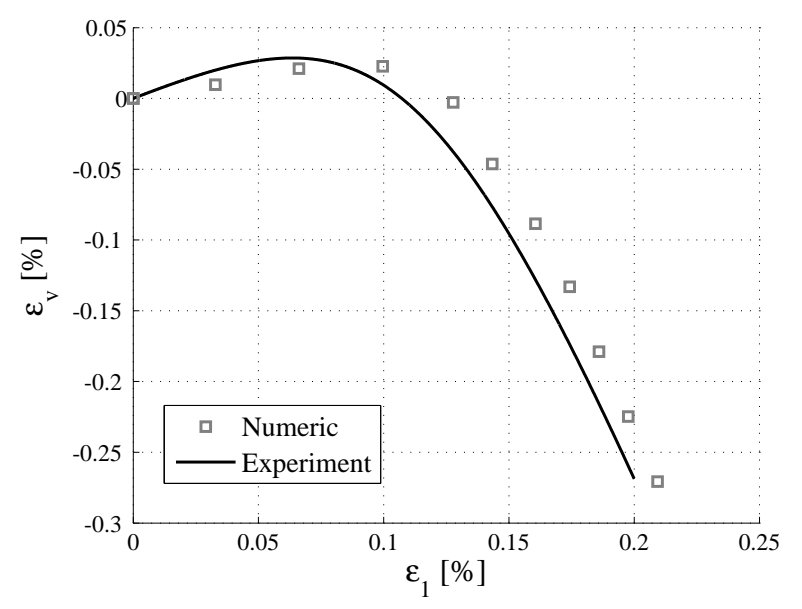

Figure 9: Comparison of monotonic simple compression simulation and experimental results, volumetric vs. axial deformation

to the activation of the boundary surface. Therefore this is a consequence of the failure and not a cause.

Since $\mathrm{p}_{c}$ is almost not decreased at failure, the cohesion at peak can be used to estimate the initial value of $\mathrm{p}_{c}$. This is true especially if the degradation rate $A_{c}$ is low.

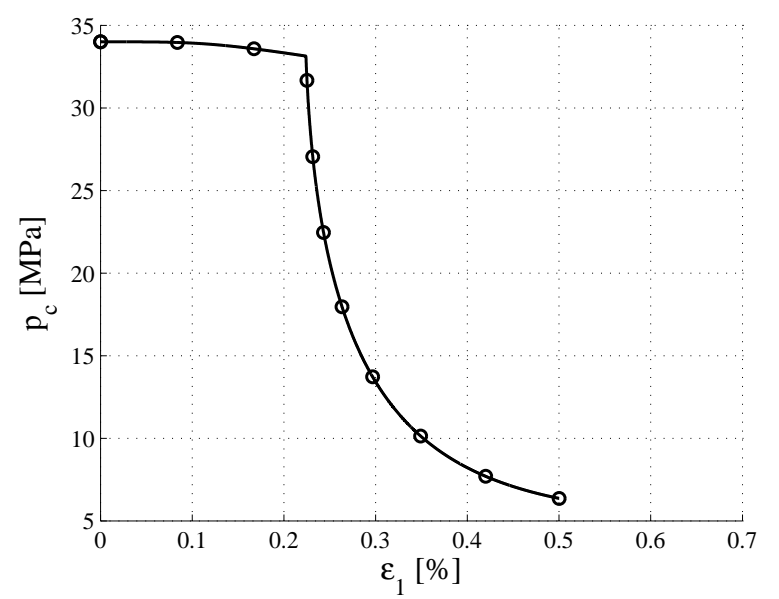

Figure 10: Evolution of the $\mathrm{p}_{c}$ internal variable during the simple compression test

It was impossible to reproduce the cyclic experiment with the same set of parameters due to the large variability of experimental results. Indeed it can be observed that monotonic test and the first compression of the cyclic test diverge in Figure 3. Results of a simulation are provided in Figure 11 assuming $\mathrm{M}^{h}=1.7, b_{0}=10^{5}, \mathrm{M}^{b}=1.6$ and $A_{c}=150$.

The experimental result exhibit a failure of the sample after almost 30 cycles. The numerical simulation leads to failure after 25 cycles. Numerical results well capture the accumulation of deformation. However the deformation is a bit too high. The difference between lateral and axial deformation is also well reproduced. The opening of the last cycles is increasing which was required.

Failure is finally reached due to the progressive alteration of the cohesion ( $\mathrm{p}_{c}$ internal variable), as depicted in Figure 12. In this case, degradation of the

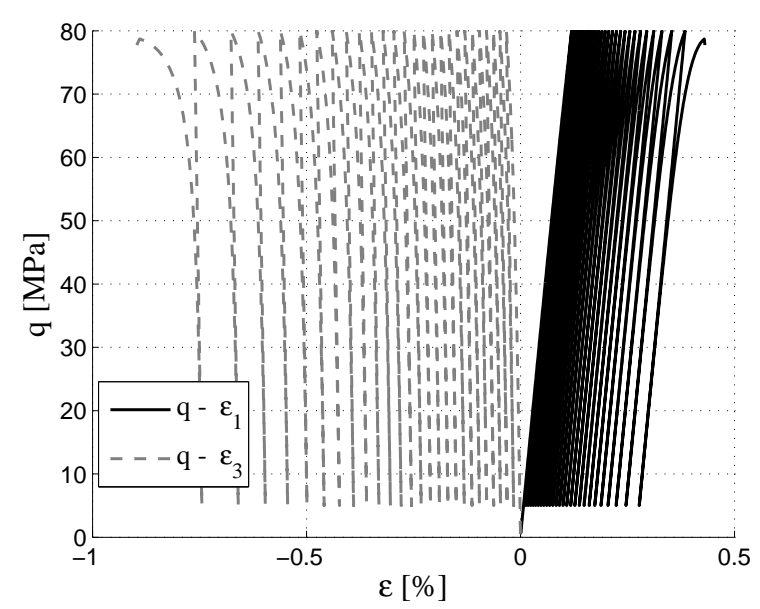

Figure 11: Comparison of cyclic simple compression simulation and experimental results (envelope only), stress deviator vs. axial deformation

cohesion is the cause of the failure. This is consistent with the most diffusion and progressive mechanism of failure. The post-peak behaviour is not modelled since the simulation is stress-driven.

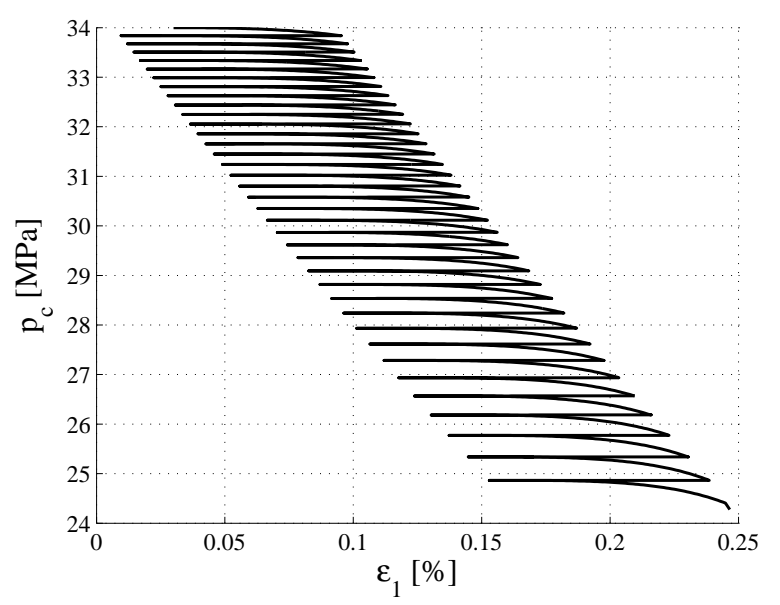

Figure 12: Evolution of the $\mathrm{p}_{c}$ internal variable during the cyclic simple compression test

The non-linear unloading part of the cycles is not reproduced. Indeed, this feature is probably due to the re-opening of crack within the sample. The crack closure phase is not captured by the model. Therefore the re-opening phase is not. However since the openingclosure of the cracks as well as friction dissipate energy, modelling this phase could be an improvement of the model.

\subsection{Brittleness and stiffness}

The brittleness of the failure depends on two parameters: $\mathrm{M}^{h}$ and the exponent $N$. Results are provided for different values of these parameters. The decohesion parameter $A_{c}$ is set to zero to simplify the analysis, i.e. there is no degradation of cohesion before failure. Both parameters modifies the plastic deformation reached at failure, as shown in Figures 13 and 14 . When $\mathbf{M}^{h}$ tends to $\mathbf{M}^{b}$, the original bounding surface concept is recovered and the yield surface tends 
towards the bounding one without reaching it, the deformation is infinite.

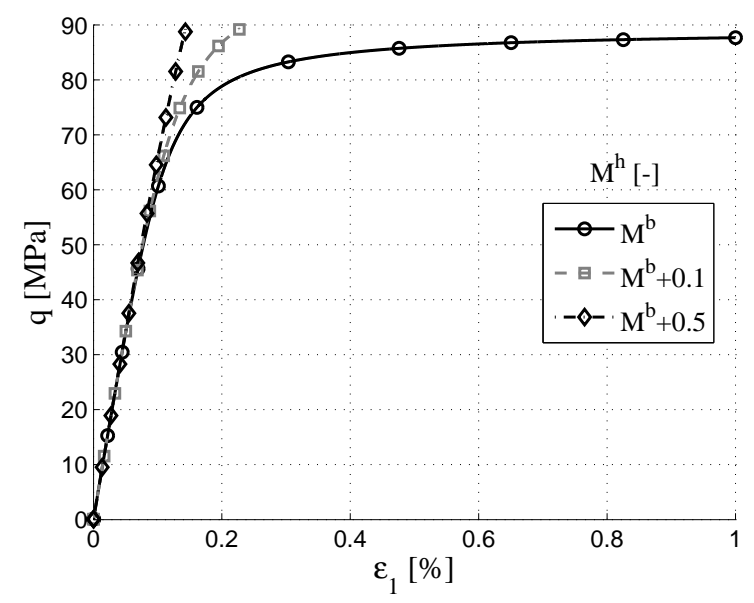

Figure 13: Influence of the $\mathrm{M}^{h}$ parameter

When only the exponent $N$ is modified, the plastic deformation increases at failure. However the deformation is always finite. This parameter also increases the divergence from linearity since the plastic multiplier is a function of $\left|\alpha-\alpha^{h}\right|^{N}$. Therefore as soon as $\left|\alpha-\alpha^{h}\right|<1$, the loss of stiffness is higher for higher exponents.

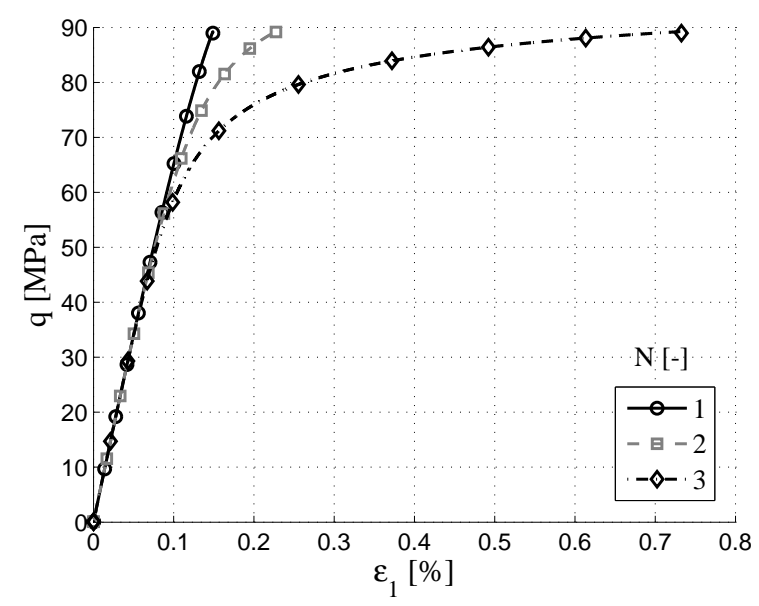

Figure 14: Influence of the $N$ parameter

The $b_{0}$ parameter directly influences the stiffness of the elastoplastic behaviour. For the present example, the elastic limit is overpassed for a stress equal to 20MPa. Divergence of the elastoplastic behaviour is more obvious for lower $b_{0}$, as shown in Figure 15.

\subsection{Dilatancy}

The parameter $A_{d}$ classically rules the plastic volumetric behaviour. It is dilative if $A_{d}<0$ and contractive otherwise as shown in Figure 16.

\subsection{Decohesion}

Parameters $\mathrm{M}^{p c}$ and $A_{c}$ regulate the degradation of the cohesion. Results of cyclic loading are provided

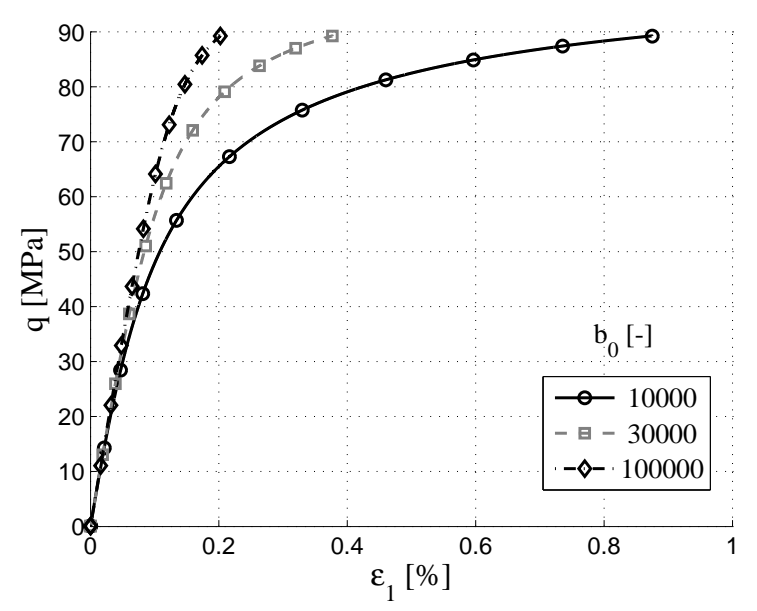

Figure 15: Influence of the $b_{0}$ parameter

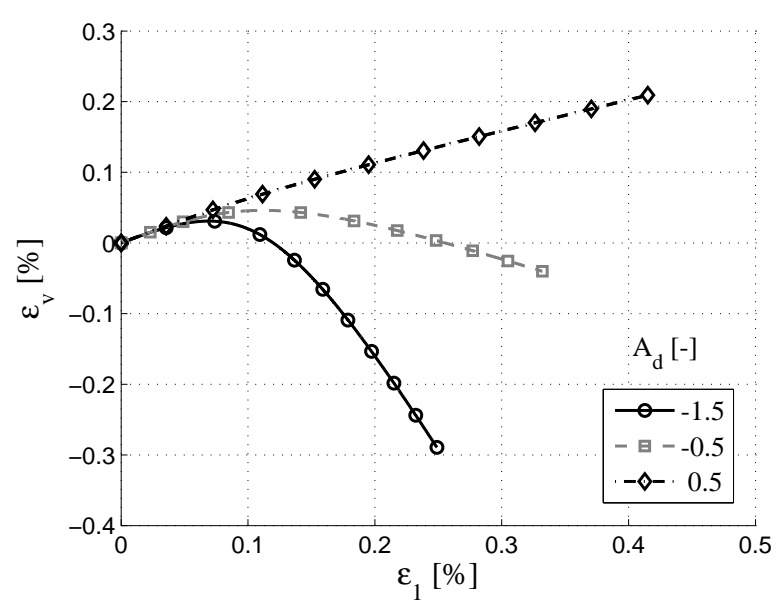

Figure 16: Influence of the $A_{d}$ parameter

in Figures 17 and 18. $\mathrm{M}^{p c}$ is a threshold beyond which degradation of the cohesion starts. Therefore the lower this value, the higher the degradation caused at each cycle. This defines the fatigue resistance of the rock.

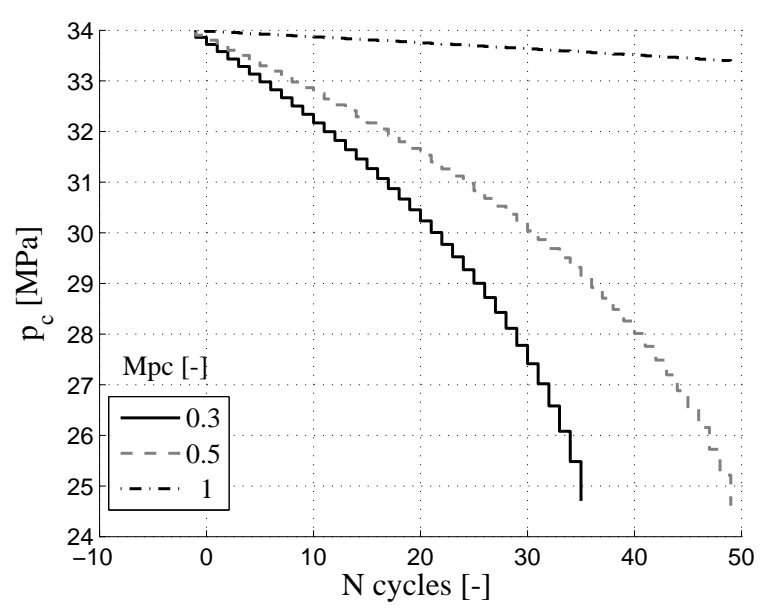

Figure 17: Influence of the $\mathrm{M}^{p c}$ parameter

The $A_{c}$ parameter rules the degradation rate of the cohesion parameter. It matters only when the stress path overcomes the degradation limit $\mathbf{M}^{p c}$. This parameter indirectly influence the number of cycles before failure that can be afford by a rock sample. 


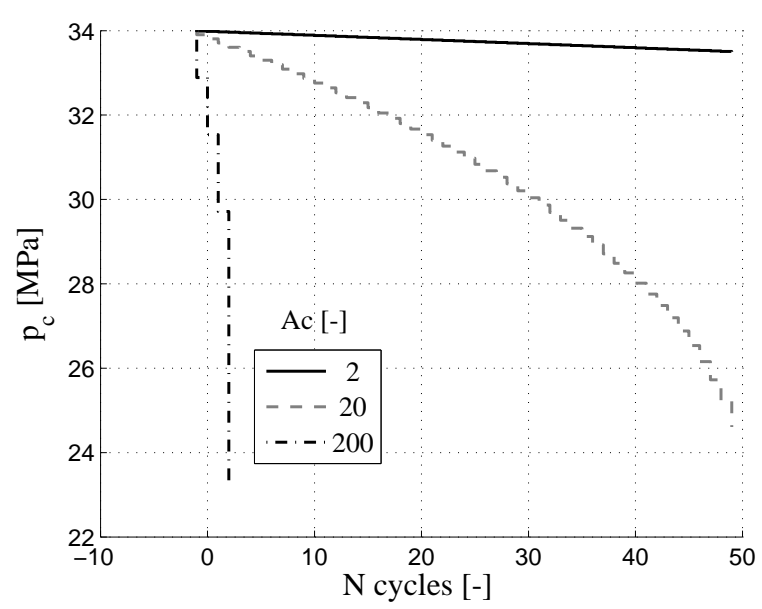

Figure 18: Influence of the $A_{c}$ parameter

\section{CONCLUSION}

This paper presents the mathematical formulation of a new model describing the cyclic behaviour of rock materials. It is based on the boundary surface framework developed for sands. It reproduce the main features of the monotonic and cyclic behaviours of rock material at low confinement,

- brittle failure of the material,

- post-peak behaviour,

- accumulation of plastic deformation upon cyclic load,

- progressive opening of the cycles,

- degradation of the cohesion,

- fatigue resistance of the material.

A comparison with experiments exhibits that the model well reproduces the monotonic results. However additional triaxial experiments should be necessary to calibrate more accurately all the parameters. The cyclic behaviour is qualitatively captured. However the simulation leads to excessive plastic deformation.

Further developments could be interesting. The damaging of elastic properties or modification of the plastic modulus may improve the model. A confinement dependency of the elastic parameter may also be introduced. The modelling of the hysteresis of the cycles could be of interest. However all of these modifications require more experimental results to be calibrated which reduces their applicability to actual projects.

\section{REFERENCES}

Attewell, P. \& W. Farmer (1973). Fatigue behaviour of rock. International Journal of Rock Mechanics and Mining Sciences 10, 1-9.
Benz, T. \& R. Schwab (2008). A quantitative comparison of six rock failure criteria. International Journal of Rock Mechanics and Mining Sciences 45, 1176-1186.

Bieniawski, Z. (1967a). Mechanism of brittle fracture of rock: PART I Theory of the fracture process. International Journal of Rock Mechanics and Mining Sciences 4, 395-406.

Bieniawski, Z. (1967b). Mechanism of brittle fracture of rock: PART II Experimental studies. International Journal of Rock Mechanics and Mining Sciences 4, 407-423.

Cai, M., P. Kaiser, Y. Tasaka, T. Maejima, H. Morioka, \& M. Minami (2004, jul). Generalized crack initiation and crack damage stress thresholds of brittle rock masses near underground excavations. International Journal of Rock Mechanics and Mining Sciences 41(5), 833-847.

Cattaneo, S. \& J. Labuz (2001). Damage of marble from cyclic loading. Journal of materials in civil engineering 13(December), 459-465.

Erarslan, N. \& D. J. Williams (2012, nov). Investigating the Effect of Cyclic Loading on the Indirect Tensile Strength of Rocks. Rock Mechanics and Rock Engineering 45(3), 327340.

Ferrero, A., M. Migliazza, \& A. Spagnoli (2009). Theoretical modelling of bowing in cracked marble slabs under cyclic thermal loading. Construction and Building Materials 23(6), 2151-2159.

Førsund, F. (2015). Hydropower economics, Volume 217. Springer.

Liu, E. \& S. He (2012). Effects of cyclic dynamic loading on the mechanical properties of intact rock samples under confining pressure conditions. Engineering Geology 125, 81-91.

Martin, C. D. (1997, oct). Seventeenth Canadian Geotechnical Colloquium: The effect of cohesion loss and stress path on brittle rock strength. Canadian Geotechnical Journal 34(5), 698-725.

Royer-Carfagni, G. \& W. Salvatore (2000, oct). The characterization of marble by cyclic compression loading: experimental results. Mechanics of Cohesive-frictional Materials 5(7), 535-563.

Simo, J. \& T. Hughes (1998). Computational inelasticity. Springer-Verlag, New-York.

Stavropoulou, M., P. Liolios, \& G. Exadaktylos (2004). Calibration of the Triaxial Hyperbolic Mohr-Coulomb Elastoplastic Model Parameters on Laboratory Rock Mechanics Tests. International Journal of Geomechanics 12(December), 618631.

Steffen, B. (2012). Prospects for pumped-hydro storage in germany. Energy Policy 45, 420-429.

Taiebat, M. \& Y. Dafalias (2008). SANISAND: simple anisotropic sand plasticity model. International Journal for Numerical and Analytical Methods in Geomechanics 32(March 2007), 915-948. 